Revue internationale P.M.E.

Économie et gestion de la petite et moyenne entreprise

\title{
Les nouveaux défis de la TPE dans les pays du Sud
}

\section{Marie-Thérèse Um-Ngouem}

Volume 19, numéro 1, 2006

URI : https://id.erudit.org/iderudit/1008492ar

DOI : https://doi.org/10.7202/1008492ar

Aller au sommaire du numéro

Éditeur(s)

Presses de l’Université du Québec

ISSN

0776-5436 (imprimé)

1918-9699 (numérique)

Découvrir la revue

Citer cette note

Um-Ngouem, M.-T. (2006). Les nouveaux défis de la TPE dans les pays du Sud. Revue internationale P.M.E., 19(1), 119-141. https://doi.org/10.7202/1008492ar

\section{Résumé de l'article}

La TPE du Sud serait-elle devenue un objet d'étude en sciences de gestion ?

Depuis quelques années, la TPE en Occident est un champ d'étude constamment exploré. Pendant ce temps, perçue comme un lieu de débrouillardise et de survie, son homologue du Sud n’a pas su susciter le même intérêt. Pourtant, le développement de la TPE concerne aujourd'hui au moins autant de personnes dans les pays du Sud que la grande entreprise. Cet article ouvre une réflexion sur la caractérisation des micro-comportements dans les TPE du Sud, en tant qu'objet spécifique d'étude. Il en souligne l'évolution, les défis actuels et la capacité à les relever. Il met l'accent, dans un souci de validité, sur la nécessaire contextualisation des outils et modèles d'analyse traditionnelle pour saisir la complexité de ces unités de production sans dénaturation. La problématique abordée devrait ainsi déboucher sur des pistes d'une véritable recherche sur ces entreprises d'un type particulier.
Ce document est protégé par la loi sur le droit d'auteur. L'utilisation des services d’Érudit (y compris la reproduction) est assujettie à sa politique d'utilisation que vous pouvez consulter en ligne.

https://apropos.erudit.org/fr/usagers/politique-dutilisation/ 
Note de recherche

\title{
Les nouveaux défis de la TPE dans les pays du Sud 1
}

Marie-Thérèse UM-NGOUEM Université de Douala

Cameroun

\section{MOTS CLÉS}

\section{TPE - Informel - Contextualisation - Dénaturation Défis - Originalité - Long terme}

\begin{abstract}
RÉSUMÉ
La TPE du Sud serait-elle devenue un objet d'étude en sciences de gestion?

Depuis quelques années, la TPE en Occident est un champ d'étude constamment exploré. Pendant ce temps, perçue comme un lieu de débrouillardise et de survie, son homologue du Sud n'a pas su susciter le même intérêt. Pourtant, le développement de la TPE concerne aujourd'hui au moins autant de personnes dans les pays du Sud que la grande entreprise. Cet article ouvre une réflexion sur la caractérisation des micro-comportements dans les TPE du Sud, en tant qu'objet spécifique d'étude. Il en souligne l'évolution, les défis actuels et la capacité à les relever. II met l'accent, dans un souci de validité, sur la nécessaire contextualisation des outils et modèles d'analyse traditionnelle pour saisir la complexité de ces unités de production sans dénaturation. La problématique abordée devrait ainsi déboucher sur des pistes d'une véritable recherche sur ces entreprises d'un type particulier.
\end{abstract}

\section{L'AUTEURE}

MARIE-ThÉRĖSE UM-NGOUEM détient un doctorat en sciences de gestion de l'Université Montesquieu, Bordeaux IV. Ancien cadre de banque, elle est maintenant enseignante à la FSEGA de I'Université de Douala au Cameroun. Ses domaines de recherche sont le financement de la petite entreprise et les systèmes financiers informels. Elle est membre de l'Unité de formation doctorale d'Économie monétaire et bancaire de l'Université de Douala et du Centre de recherche en entreprise familiale de I'Université Montesquieu Bordeaux IV. Courriel:<ngouem@yahoo.fr>.

1. Je tiens à remercier tout particulièrement les lecteurs connus et anonymes pour la rigueur et la précision de leurs critiques, commentaires et observations.

(C) 2006 - Presses de l'Université du Québec

Édifice Le Delta I, 2875, boul. Laurier, bureau 450, Québec, Québec G1V 2M2 • Tél.: (418) 657-4399 - www.puq.ca

Tiré de: Revue internationale P.M.E., vol. 19, $\mathrm{n}^{\circ}$ 1, sous la direction de Louis Raymond • PME1901N

Tous droits de reproduction, de traduction et d'adaptation réservés 


\begin{abstract}
Does the very small enterprise in the developing countries belong to the scientific management of the firm?

For several years, the very small enterprise in the western world is a field of study which is constantly explored. During this time, perceived as a means of survival, the interest of the Minor Enterprise in developing countries is not underlined enough. However, nowadays, the development of minor enterprises concerns at least so many persons in developing countries as big enterprises. The purpose of the paper is to encourage a brainstorming on micro-behaviour of these enterprises as a specific conceptual framework. It highlights its evolution, present challenges and the capacity to overcome them. As a way of validity, it stresses the necessary contextualisation of tools and traditional analysis models in order to understand the complexity of these unities of production without changing their nature. The problematic approached should thus come out with a real field of research for these particular enterprises.
\end{abstract}

\title{
RESUMEN
}

¿La pequenita empresa del Sur habria formado parte de la literatura cientifica de la empresa?

Desde muchos años la pequenita empresa en los paises desarollados es un tema muy estudiado, lo que no es lo mismo en los paises subdesarollados. Sin embargo, hoy dias el desarollo de la pequenita empresa a lo menos consta con tantas personas como la empresa grande en los paises del Sur. El estudio hace hincapie en la adaptacion de modelos de analisis para comprender lo complejo de estas empresas. El articulo este Ilama a una reflexion en cuanto a la manera de acabar con su desafio. El problema planteado deberia desembocar en un verdadero estudio sobre estas empresas particulares.

\section{ZUSAMMENFASSUNG}

Seit einigen Jahren ist das Kleinstunternehmen in der westlichen Welt schon Gegenstand von Untersuchungen. In der gleichen Zeit konnte das Kleinstunternehmen in südlichen Ländern nicht dasselbe Interesse der Forschenden auf sich ziehen. Durch die Entwicklung von Kleinstunternehmen in den Ländern der südlichen Hemisphäre sind heute jedoch mindestens genauso viele Personen davon betroffen wie in grossen Unternehmen. Dieser Artikel eröffnet die Diskussion über die Charakterisierung der Mikro-Verhaltensweisen in Kleinstunternehmen des Südens und betrachtet sie dabei als spezifische Studienobjekte. Der Artikel unterstreicht dabei in besonderem Masse die derzeitigen Herausforderungen und die Fähigkeiten, die Herausforderungen zu meistern. Betont wird überdies, die Werkzeuge und Modelle traditioneller Analysemethoden anzupassen, damit die Komplexität von diesen Unternehmen, ohne Verlust der Eigenart, erfasst werden können. Die angesprochenen Probleme sollten hernach zu einem echten Forschungszweig für diese speziellen Unternehmen führen.

Revue internationale P.M.E., vol. 19, $\mathrm{n}^{\circ} 1,2006$

(C) 2006 - Presses de l'Université du Québec

Édifice Le Delta I, 2875, boul. Laurier, bureau 450, Québec, Québec G1V 2M2 - Tél.: (418) 657-4399 - www.puq.ca

Tiré de: Revue internationale P.M.E., vol. 19, no 1, sous la direction de Louis Raymond • PME1901N

Tous droits de reproduction, de traduction et d'adaptation réservés 


\section{Introduction}

La montée en puissance depuis le début des années 1980 des petites entreprises en Occident semble être attachée au coup de fouet que celles-ci donnent au tissu économique après le passage de l'ouragan de la crise industrielle des années 1970. Celles-ci apparaissent alors comme un instrument de régénération des économies en période difficile (Julien et Marchesnay, 1996). La dynamique ainsi reconnue à ce type d'entreprise est à la base de la réflexion sur le rôle de la TPE dans les économies du Sud confrontées au problème du développement et de la lutte contre la pauvreté. Il est cependant de la TPE comme de la PME ou de l'entreprise en général, c'est-à-dire un concept difficile à définir. D'une manière générale, deux principaux types de critères permettent de l'appréhender.

Un premier aspect définit la TPE à partir de critères quantitatifs. Il se rapporte essentiellement au nombre d'employés, à l'investissement en capital, au chiffre d'affaires et au total du bilan. Souvent, on les restreint aux deux premiers.

Du point de vue des effectifs, la TPE serait cette entreprise où le nombre de salariés ne dépasse pas 10 à 20 personnes, constituées de travailleurs non salariés (Ferrier, 2002). Du point de vue du capital, l'investissement initial y est faible et généralement la technologie incorporée à l'outil de production est peu sophistiquée.

Ces critères quantitatifs offrent plus de facilités d'identification mais posent un certain nombre de problèmes. C'est le cas, par exemple, du sens même à donner aux effectifs qui peuvent être stables ou instables, concerner des salariés temporaires ou permanents... Il en est de même des actifs engagés dans la production, de leur évaluation, à cause de la question de la qualité des données statistiques, de leur divergence quand on passe d'une source à une autre. Pour atténuer ces insuffisances, on leur adjoint des critères d'une autre nature.

Un deuxième aspect de la définition permet ainsi de privilégier les critères qualitatifs. Il s'agit essentiellement de la propriété du capital, du mode de financement, des styles de gestion où les logiques d'actions individuelles jouent un rôle essentiel comme c'est le cas pour l'art et l'artisanat en particulier (Loup, 2003). Dans l'ensemble des cas, le propriétaire se confond à l'entreprise et le problème de l'identification ne se pose pas. Mais il reste à résoudre celui des modes d'organisation et de management.

Ces critères quantitatifs et qualitatifs plus complémentaires que concurrents permettent ensemble de caractériser la TPE, souvent en la distinguant de la PE et de la PME. Seulement, s'ils tentent de circonscrire la TPE de manière générale, ils ne précisent pas encore véritablement la spécificité de la TPE du Sud. Or, celle-ci semble avoir un statut particulier. Elle est essentiellement une très petite entreprise souvent informelle, c'est-à-dire qui n'a pas une existence juridique propre mais

Revue internationale P.M.E., vol. 19, nº 1, 2006

(C) 2006 - Presses de l'Université du Québec

Édifice Le Delta I, 2875, boul. Laurier, bureau 450, Québec, Québec G1V 2M2 • Tél.: (418) 657-4399 - www.puq.ca

Tiré de: Revue internationale P.M.E., vol. 19, no 1, sous la direction de Louis Raymond • PME1901N

Tous droits de reproduction, de traduction et d'adaptation réservés 
qui devient de plus en plus formelle. Dans ce dernier cas, sa formalisation est une condition de survie et d'accès aux opportunités que lui offre un environnement où l'État fait de plus en plus de la place aux activités privées.

En tant qu'entreprise informelle, il est important de la resituer dans son contexte. En effet, parmi les définitions formelles qui existent de l'entreprise informelle, celle adoptée par la $\mathrm{XV} \mathrm{V}^{\mathrm{e}}$ Conférence internationale des Statisticiens du travail retient comme principal critère de définition l'absence d'un statut juridique et le non-enregistrement administratif. Du point de vue de l'analyse, l'influence du droit conduit à faire des contrats un des fondements des relations dans et entre les entreprises. Elle fonde des rapports «sécurisés», c'est-à-dire gérables en cas de conflits. Barredy (2002) appréhende la structure juridique comme un instrument de développement de l'entreprise. Définie par le droit des sociétés, celle-ci détermine les conditions de fonctionnement, l'organisation explicite, les responsabilités des acteurs, leurs droits et leurs obligations. Or, la TPE ne vit pas en autarcie; elle doit fonctionner aux côtés d'unités formelles. Et la permanence d'une économie à double vitesse peut engendrer des conflits dans le système de production. Lautier (1994) cite à ce sujet l'exemple des commerçants ghanéens et sénégalais dont les gouvernements des pays d'accueil, respectivement le Nigeria et la Mauritanie, ont décidé à un moment d'interdire l'activité.

On a pu constater que cet aspect de l'informel n'est pas une exclusivité de ces pays, dans la mesure où on la retrouve ailleurs. Marchesnay (2003) souligne qu'au Japon il n'existe pas de statistiques pour les petites entreprises de moins de sept salariés et qu'une situation semblable a été observée dans d'autres économies avancées. Cette perception juridique est donc incomplète pour appréhender la TPE du Sud. Une définition plus large et plus opérationnelle du secteur informel reconnaît cette inexistence juridique mais lui associe d'autres critères quantitatifs et qualitatifs dont la taille et la nature des activités, le caractère anticyclique du développement, l'absence de séparation entre capital et travail essentiellement (Tidjani, 2003). L'ensemble de ces critères semblent mieux caractériser la TPE du Sud qu'ils placent dans une logique de survie.

L'analyse devrait cependant être plus nuancée s'agissant de cette logique de survie et de son appartenance exclusive au secteur informel; car la TPE n'a pas un caractère homogène. Si sa petitesse est incontestable, sa forme est déterminée par la nature des besoins, le secteur et les objectifs de l'activité, par la formation à la base, puis par le mode d'organisation et de gestion qui en résulte. La petite activité marchande et l'artisanat qui semblent la représenter en sont la forme traditionnelle. La TPE traditionnelle informelle se définit ainsi par des effectifs se réduisant à un ou deux employés, apprentis et souvent membres de la famille, rémunérés plus ou moins en nature pour une formation sur le tas. L'investissement initial en capital est relativement faible et provient en général des ressources personnelles du proprié-

Revue internationale P.M.E., vol. 19, $\mathrm{n}^{\circ} 1,2006$

(C) 2006 - Presses de l'Université du Québec

Édifice Le Delta I, 2875, boul. Laurier, bureau 450, Québec, Québec G1V 2M2 • Tél.: (418) 657-4399 - www.puq.ca

Tiré de: Revue internationale P.M.E., vol. 19, no 1, sous la direction de Louis Raymond - PME1901N

Tous droits de reproduction, de traduction et d'adaptation réservés 
taire fondateur, de la famille (Saporta, 2002), des amis, de l'épargne des circuits informels de financement (Gnansounou, 1992; Hernandez, 1997; Um-Ngouem, 1996). L'aspect dominant est que l'organisation et la gestion sont quasi inexistantes et se font au jour le jour, en l'absence de toute «action marketing» sous quelque forme que ce soit. La modicité des ressources, une technologie rudimentaire et la faible organisation de la gestion facilitent l'entrée dans le secteur, en même temps qu'elles le prédestinent à une grande précarité et à des objectifs de court terme. Mais la TPE ne devrait pas exister uniquement sous cette forme.

Actuellement, un nouveau type de TPE semble émerger. Au départ, il n'est ni totalement formel ni totalement informel et peut être rapproché de la qualification d'informel moderne que lui donne Niang $(1991)^{2}$. Mais de plus en plus les exigences de son environnement l'oblige à se formaliser davantage de manière à bénéficier de différentes initiatives et actions de promotion ou d'appui au développement (Um-Ngouem, 1996). Des efforts de théorisation des comportements, en ce qui concerne cette évolution, sont observés depuis quelque temps (Saporta et Kombou, 2000; Tidjani, 2003). En effet, la privatisation de nombreuses entreprises $\mathrm{du}$ portefeuille de l'État bouleverse totalement l'environnement en faisant plus de place au secteur privé et, partant, à la TPE, socle de l'économie. Les critères qualitatifs et quantitatifs précédents, s'ils peuvent donner un aperçu de ce qu'est la TPE traditionnelle, apparaissent insuffisants pour souligner l'intérêt particulier de la TPE informelle ou formelle moderne.

Les effectifs selon la nouvelle tendance sont un peu plus étoffés et compris entre 1 et 10 employés (Rofe, 1998; Ntsama et al., 1999; Srinivasan et Mammen, 2002), constitués pour l'essentiel d'apprentis et d'une main-d'œuvre familiale (BIT, 1972; Hernandez, 1997). L'activité se diversifie progressivement autour d'opérations de production, d'artisanat d'art, de services et de commerces. L'investissement initial, bien que constitué d'un petit équipement et d'un outillage simple, est plus important quand on passe d'opérations de services, de commerces, à la production manufacturière. Si on devait la chiffrer, sa valeur pourrait être estimée pour un pays comme le Cameroun, entre 1 et 5 millions de francs CFA (Ntsama, Um-Ngouem et Abessolo, 2003; $\mathrm{FNE}^{3}$, 1999), entre 0,5 et 2,5 millions de roupies, soit en francs CFA entre 6,3 et 31,5 millions, dans un pays comme l'Inde (Marwaha, 2002). Cette différence dans les échelles de mesures confirme une fois de plus l'hétérogénéité du groupe. La technologie incorporée à l'outil de production reste généralement peu sophistiquée. Du point de vue de la dimension humaine, l'entrepreneur formé sur le tas ne rend plus compte des besoins et des compétences requises. Le nouveau contexte productif est devenu celui d'un entrepreneur technicien, ingénieur,

2. Cité par Tidjani (2003).

3. Le Fonds national de l'emploi au Cameroun (FNE) a pour mission de promouvoir l'auto-emploi.

Revue internationale P.M.E., vol. 19, n 1, 2006

(C) 2006 - Presses de l'Université du Québec

Édifice Le Delta I, 2875, boul. Laurier, bureau 450, Québec, Québec G1V 2M2 • Tél.: (418) 657-4399 - www.puq.ca

Tiré de: Revue internationale P.M.E., vol. 19, no 1, sous la direction de Louis Raymond • PME1901N

Tous droits de reproduction, de traduction et d'adaptation réservés 
mécanicien..., doté de connaissances théoriques et pratiques dans son champ de spécialité. Une activité mieux organisée, un investissement, des objectifs conséquents et plus encore une bonne formation deviennent des gages de réussite. La formation en particulier représente actuellement l'une des garanties, notamment pour les entrepreneurs qui postulent pour des financements auprès d'organismes privés ou publics d'appui à la petite entreprise. De nouvelles exigences s'expriment désormais en termes de formalisation, de réactivité, d'amélioration du service, de flexibilité, de créativité, de rentabilité économique, requérant de l'originalité et une implication dans le long terme.

C'est à travers ce nouveau prisme que la TPE du Sud doit être lue désormais; car elle semble avoir évolué ou être en voie de le faire. Ce potentiel de transformation, tributaire du profil de l'entrepreneur, s'impose à tous et relance le débat sur la question de l'entrepreneur, sur l'importance de la contextualisation de son activité et sur les risques de dénaturation si ce contexte n'est pas pris en compte.

La littérature sur la très petite entreprise et le comportement de l'entrepreneur contribue ainsi à la démythification du capitalisme managérial, mythe de la grande taille et des économies d'échelle, mythe également de la hiérarchie comme condition de stabilité et de sécurité (Torrès, 1998, 2003; Ferrier, 2002; Marchesnay, 2003). Elle doit désormais davantage se pencher sur la spécificité des comportements dans la TPE du Sud et sur leur possible mutation. Notre étude se propose, dans une approche critique d'une analyse parcellaire de la TPE, d'expliquer les changements de comportement dans la TPE du Sud. Elle s'appuie sur le modèle interactionniste du comportement de Lewin $(1946,1951)$. L'objectif est d'aboutir à un cadre d'analyse qui met d'abord en lumière les grands défis auxquels la TPE du Sud est confrontée puis qui souligne, dans une dynamique de rupture avec l'acteur passif, l'importance du rôle et du profil du dirigeant propriétaire, comme moyens majeurs pour relever les défis qui sont les siens.

Notre réflexion s'articule ainsi en deux principaux points:

- la TPE du Sud: la remise en cause de l'acteur passif;

- la TPE du Sud: l'affirmation d'un statut nouveau.

\section{La TPE du Sud: la remise en cause de l'acteur passif}

La remise en cause de l'acteur passif résume l'importance des défis auxquels fait face la TPE du Sud. Ces défis sont principalement la maîtrise de l'entreprise spontanée, puis l'émergence d'une TPE plus flexible en accord avec l'évolution de l'environnement.

Revue internationale P.M.E., vol. 19, nº 1, 2006

(C) 2006 - Presses de l'Université du Québec

Édifice Le Delta I, 2875, boul. Laurier, bureau 450, Québec, Québec G1V 2M2 • Tél.: (418) 657-4399 - www.puq.ca

Tiré de: Revue internationale P.M.E., vol. 19, no 1, sous la direction de Louis Raymond - PME1901N

Tous droits de reproduction, de traduction et d'adaptation réservés 


\subsection{La maîtrise de la génération spontanée}

La génération spontanée est cette TPE traditionnelle qui surgit de nulle part, à partir de tout et de rien. Sa maîtrise constitue le premier défi de la TPE dans les pays du Sud. Elle consiste en l'identification de ses faiblesses qui se résument à une faible prise en compte du risque et un pilotage à vue. L'objectif est de parvenir à les corriger au fur et à mesure que les changements de l'environnement l'imposent.

\subsubsection{La question du risque}

Le facile accès à la création de l'entreprise dans les TPE semble justifier l'entrée dans le secteur informel ${ }^{4}$. Celui-ci est alors représenté essentiellement par de petites activités de routine ne nécessitant aucune formalité administrative, aucun niveau particulier de ressources financières, matérielles et humaines. Il s'agit pour la plupart d'activités domestiques de proximité, d'artisans isolés, du petit commerce et service. Il se constitue autour d'une classe d'entrepreneurs spontanés, formés sur le tas et caractérisés par une évaluation sommaire de leurs risques.

La création d'une entreprise est, en effet, une «aventure». L'entrepreneur, d'après Knight (1921) est celui qui doit prendre des décisions dans un contexte de grande incertitude. Le profit de l'entrepreneur est donc une récompense du risque et de l'incertitude entretenue par son action. Précisément, la prise de conscience que le risque existe est le point de départ de la création d'entreprise, le risque étant considéré comme le degré de probabilité attaché à l'échec. Les risques auxquels s'expose l'entrepreneur, dès lors, sont multiples. L'analyse en présente plusieurs types que l'on peut regrouper en risques financiers, stratégiques et opérationnels. Créer une entreprise suppose ainsi l'identification du risque, l'appréhension des facteurs d'aggravation de celui-ci. La prévention, la prévision, l'évaluation du risque apparaissent alors comme des outils de gestion et de maitrise du risque. La maturation de l'idée, une étude de faisabilité, l'évaluation des capacités techniques, financières, humaines, une vision à long terme de l'entreprise en sont des indicateurs. Elles sont en même temps des conditions de la réalisation du profit, premier véritable enjeu financier pour toute entreprise. Car la fonction financière est inséparable de la création de valeur (Caby et Hirigoyen, 2001).

La TPE traditionnelle semble fonctionner sans totalement intégrer ces données. Les ressources financières sont modiques et tirées de sources informelles

4. La formalisation a en effet un coût qui se traduit dans les charges financières mais aussi dans le temps nécessaire pour satisfaire aux formalités et règles de constitution des sociétés. Ces coûts sont qualifiés par De Soto (1994) de coûts de durabilité de la légalité. Hernandez (1997), en prenant l'exemple du contexte péruvien étudié par l'Institut Liberté et Démocratie du Pérou (ILD), relève, quant à lui, le coût financier excessif relié à l'installation d'une petite industrie.

Revue internationale P.M.E., vol. 19, n 1, 2006

(C) 2006 - Presses de l'Université du Québec

Édifice Le Delta I, 2875, boul. Laurier, bureau 450, Québec, Québec G1V 2M2 • Tél.: (418) 657-4399 - www.puq.ca

Tiré de: Revue internationale P.M.E., vol. 19, no 1, sous la direction de Louis Raymond • PME1901N

Tous droits de reproduction, de traduction et d'adaptation réservés 
éparses, avec des coûts implicites qui peuvent être supérieurs au profit attendu (Um-Ngouem, 1997). De même, la population employée est astreinte à un faible niveau d'une rémunération allouée quelques fois en nature. La relation d'emploi est rarement une relation marchande ou contractuelle et résulte souvent de liens de parenté (Torrès, 2001). Les relations de travail sont dominées par des rapports de dépendance et d'allégeance et peuvent aussi apparaitre comme un sujet de désaccord même dans l'hypothèse d'une réussite sur le plan économique (Saporta, 2002). Les risques financiers, opérationnels et stratégiques sont donc insuffisamment appréhendés.

L'omission de ces fondations de la «démarche» de création de TPE dans les pays du Sud les inscrit dans une logique de «débrouillardise», de survie et de création de revenus immédiats. Mais cela ne signifie pas que le risque n'est pas présent dans l'esprit de l'entrepreneur, ni que ce dernier ignore l'intérêt du profit. Tant s'en faut. Le vendeur à la sauvette ne vend pas en dessous de son prix d'achat. Il va à l'assaut du client à l'entrée des magasins et dans tous les lieux de grande affluence, essayant de vendre au même prix et parfois à un prix supérieur à celui de l'entreprise formelle qui, elle, supporte des charges fixes. Il n'ignore donc pas le risque. Il le mesure aussi à travers le niveau du chiffre d'affaires et du bénéfice qui en découle. Par contre, il ne va pas au bout du processus et confond facilement recettes et bénéfices pour faire face à ses besoins courants. De ce point de vue, il semble le minimiser en ne développant pas d'actions durables et intrinsèques sur les prix, les produits, le financement, le capital humain... La précarité de l'activité et la menace de disparition sont permanentes, tant l'avenir est incertain. L'attrait du secteur est réel mais pourrait être accru par une meilleure identification et gestion du risque. Sa spécificité ne peut être maîtrisée et valorisée que s'il naît un véritable esprit d'entreprise qui détermine comment le risque sera appréhendé et pris en compte dans le processus de décision. C'est dans ce contexte que le pilotage à vue apparaît comme une autre source irréductible d'incertitude.

\subsubsection{Les limites du pilotage à vue}

En effet, maîtriser la génération spontanée, c'est aussi prendre conscience des limites d'un pilotage à vue. La préoccupation de création de revenus immédiats qui se manifeste dans le recours constant à ce secteur ne témoigne guère d'objectifs et de moyens clairement définis. Dans la petite activité marchande, les conditions de l'activité restent hasardeuses, sans de véritables règles de gestion, sans aucun support physique de l'information comptable et financière, sans un horizon connu (Tidjani, 2003). Or, l'évolution de l'entreprise n'est ni libre ni aléatoire. Elle suit, selon la thèse évolutionniste, un sentier déterminé par la nature des compétences accumulées. Au-delà du caractère déterministe de cette thèse, il y a un aspect dynamique. Il réside dans la capacité pour l'entreprise à définir une trajectoire et

Revue internationale P.M.E., vol. 19, $\mathrm{n}^{\circ} 1,2006$

(C) 2006 - Presses de l'Université du Québec

Édifice Le Delta I, 2875, boul. Laurier, bureau 450, Québec, Québec G1V 2M2 • Tél.: (418) 657-4399 - www.puq.ca

Tiré de: Revue internationale P.M.E., vol. 19, no 1, sous la direction de Louis Raymond - PME1901N

Tous droits de reproduction, de traduction et d'adaptation réservés 
à induire les changements qui s'imposent à elle, qu'il s'agisse de la gestion interne ou des politiques de marché. En effet, ces choix se manifestent dans un certain nombre de traits (Marchesnay, 1997):

- l'esprit d'innovation ou de création qui pousse l'entrepreneur, face à l'incertitude, à transformer son environnement et non pas à s'y adapter comme cela a souvent été le cas pour le «sauveteur» dans les premières générations de TPE dans les pays du Sud;

- l'esprit d'organisation et de coordination de l'activité qui exige, entre autres, l'acquisition et l'exploitation de l'information. La forte composante humaine et familiale dans la vie de la TPE et les liens sociaux qu'elle suppose (Basly, 2002; Hirigoyen, 2002) devant constituer un atout dans le développement des ressources informationnelles;

- l'assomption des divers risques et plus encore du risque financier qui s'exprime dans le fait d'engager parfois l'intégralité de ses moyens financiers dans son affaire, dans l'espoir d'en tirer un profit conséquent.

Rompre avec une économie de subsistance, c'est reconnaître à l'innovation, à l'organisation, à l'assomption du risque un rôle moteur en tant qu'outils de gestion et de pilotage contrôlé de l'entreprise. L'entreprise informelle traditionnelle apparaît comme le modèle type de l'entreprise fragile. Émanation d'une génération spontanée, elle n'évalue pas suffisamment le risque et ne se fixe pas d'horizon dans le temps. Pour sa pérennité et son attrait, la prise de conscience de ces aspects est fondamentale. Elle n'est pas encore totale, mais on observe de plus en plus l'apparition d'un type de TPE plus élaboré, qui se construit progressivement, à la faveur des mutations de l'environnement. Le nombre croissant d'entrées dans le secteur en souligne bien le potentiel, comme le révèle le tableau 1.

Revue internationale P.M.E., vol. 19, n 1, 2006

(C) 2006 - Presses de l'Université du Québec

Édifice Le Delta I, 2875, boul. Laurier, bureau 450, Québec, Québec G1V 2M2 • Tél.: (418) 657-4399 - www.puq.ca

Tiré de: Revue internationale P.M.E., vol. 19, no 1, sous la direction de Louis Raymond - PME1901N

Tous droits de reproduction, de traduction et d'adaptation réservés 
TABLEAU 1

Évolution de la population active employée dans le secteur informel dans 20 pays du Sud de 1975 à 1998 (en \%)

\begin{tabular}{|c|c|c|c|c|c|}
\hline Pays $\longrightarrow$ Années & 1975 & 1980 & 1985 & 1990 & 1998 \\
\hline Bénin & 37 & 42 & 60 & 67 & 75 \\
\hline Burkina-Faso & 40 & 45 & 62 & 65 & 74 \\
\hline Cameroun & 33 & 37 & 55 & 63 & 72 \\
\hline Colombie & 31 & 39 & 57 & 62 & 68 \\
\hline Côte-d'Ivoire & 27 & 31 & 51 & 59 & 68 \\
\hline Ghana & 38 & 42 & 63 & 68 & 73 \\
\hline Haïti & 41 & 47 & 66 & 71 & 80 \\
\hline Inde & 37 & 42 & 55 & 62 & 69 \\
\hline Kenya & 33 & 39 & 61 & 65 & 70 \\
\hline Madagascar & 38 & 43 & 63 & 68 & 72 \\
\hline Mali & 40 & 45 & 61 & 66 & 74 \\
\hline Mexique & 28 & 34 & 50 & 53 & 60 \\
\hline Nigeria & 38 & 43 & 59 & 63 & 70 \\
\hline Niger & 42 & 46 & 64 & 69 & 75 \\
\hline Philippines & 38 & 43 & 64 & 70 & 71 \\
\hline République du Congo & 35 & 42 & 65 & 73 & 82 \\
\hline Sénégal & 29 & 35 & 53 & 64 & 71 \\
\hline Thaillande & 21 & 32 & 41 & 47 & 54 \\
\hline Togo & 43 & 48 & 64 & 69 & 74 \\
\hline Vietnam & 37 & 40 & 53 & 61 & 69 \\
\hline
\end{tabular}

Source: Kengne et Metton (2002).

Ce tableau illustre bien l'importance croissante du secteur informel. Son poids a presque doublé en 23 ans. Cette évolution laisse entrevoir de bonnes perspectives pour les acteurs et confirme l'existence d'un potentiel sur le plan industriel, commercial et des services avec la tendance actuelle de la TPE.

\subsection{La tendance actuelle de la TPE}

Jusqu' au milieu des années 1980, l'État a joué un rôle déterminant dans l'animation de la vie économique et industrielle de la plupart de ces pays. Il en a même souvent été l'un des grands acteurs sinon le principal, dans la montée des grandes unités de production créatrices d'emplois. Grâce au recul de ce poids, la TPE prend une autre envergure avec les occasions d'affaires qui s'offrent à l'entreprises privée. Elle doit pour cela faire face à de nombreux défis, défi de l'originalité, défi de l'implication à long terme principalement, pour se frayer une place et «sortir de l'ignorance».

Revue internationale P.M.E., vol. 19, $\mathrm{n}^{\circ} 1,2006$

(C) 2006 - Presses de l'Université du Québec

Édifice Le Delta I, 2875, boul. Laurier, bureau 450, Québec, Québec G1V 2M2 • Tél.: (418) 657-4399 - www.puq.ca

Tiré de: Revue internationale P.M.E., vol. 19, no 1, sous la direction de Louis Raymond • PME1901N

Tous droits de reproduction, de traduction et d'adaptation réservés 


\subsubsection{Le défi de l'originalité}

Les modèles théoriques suggèrent à toute entreprise de favoriser une dynamique entrepreneuriale fondée sur l'inventivité dans le processus de décision, dans la gestion des variables clés, dans le mode de coordination et de contrôle de l'évolution de l'activité; celle-ci tire ainsi mieux parti des transformations possibles de l'environnement. Deux étapes importantes dans cette évolution doivent être soulignées, soit le positionnement des produits et la spécificité de l'organisation.

La première étape dans ce défi est la création d'un marché réel ou potentiel facilitée par le recul du rôle économique de l'État, réalisée surtout dans une adaptation à la demande par la flexibilité du système des prix. Les prix ainsi que le degré d'élaboration du produit ou du service s'adaptent ici au niveau du revenu de la clientèle. Cette action leur confère un avantage économique distinctif malgré leur faiblesse technologique et une qualité moyenne des produits. Elles tirent profit de la situation grâce à beaucoup d'ingéniosité pour assurer un rapport qualité-prix le plus avantageux, dans un effort de persuasion du consommateur. Il s'agit par conséquent de rechercher l'ensemble des moyens destinés à faciliter les échanges de biens et services pour stimuler l'achat des consommateurs, déterminer les besoins et désirs des clients, les prévoir, révéler les besoins latents et proposer des produits ou services qui leur conviennent. Au total, en exerçant une pression de l'offre et en créant la rareté aux yeux du client, elles peuvent offrir un produit unique. Cette démarche dans la nouvelle TPE participe d'une action marketing semblable à celle décrite par Cocula (2002).

La deuxième étape de ce défi concerne l'originalité du mode d'organisation de l'activité. L'idée que les petites entreprises ne sont pas organisées est essentiellement vérifiée du point de vue de l'intervention de l'État pour structurer le secteur. Mais il est de moins en moins vrai que l'organisation de l'activité y est totalement absente.

Des études monographiques sur des TPE informelles au Cameroun ont révélé une structuration du secteur à partir d'unités «spécialisées», distinctes et complémentaires dans de petits métiers maîtrisés individuellement dans le bâtiment, la confection, les garages de réparation et d'entretien d'automobiles (Um-Ngouem, 1996). L'organisation n'est pas forcément géographique; elle est principalement fonctionnelle. Chaque spécialité intervient ponctuellement et joue alors un rôle particulier dans la «chaîne» des services ou des produits. Les compétences distinctives sont sollicitées lorsque le besoin se fait sentir, pour la satisfaction d'un même client. C'est une forme d'innovation qui vise à améliorer la qualité d'une production individualisée et de proximité. Elle permet à la TPE d'être compétitive et de développer ses parts de marché. Cette technique est centrée sur l'individualisation du produit, sur la flexibilité, sur une création de valeur ajoutée réelle, tributaire du caractère unique du produit et du client. Elle peut alors s'ouvrir sur un horizon long.

Revue internationale P.M.E., vol. 19, $\mathrm{n}^{\circ}$ 1, 2006

(C) 2006 - Presses de l'Université du Québec

Édifice Le Delta I, 2875, boul. Laurier, bureau 450, Québec, Québec G1V 2M2 • Tél.: (418) 657-4399 - www.puq.ca

Tiré de: Revue internationale P.M.E., vol. 19, no 1, sous la direction de Louis Raymond • PME1901N

Tous droits de reproduction, de traduction et d'adaptation réservés 


\subsubsection{Le défi de l'implication à long terme}

L'inventivité et la rentabilité comme clés de lecture de l'efficience de l'entreprise ne s'observent que dans la durée, car toute performance se mesure par rapport à un temps de réalisation (Batsch, 1997). Parmi les choix possibles sur le long terme peut s'inscrire le double avantage que présentent, d'une part, la réduction de coûts et l'obligation de polyvalence du propriétaire dirigeant, d'autre part.

Du point de vue de la réduction des coûts, on peut relever l'intérêt de limiter ceux relatifs au temps de l'information et de la coordination d'une activité faisant intervenir plusieurs spécialités. La proximité due au regroupement par affinité ethnique ou par mimétisme favorise la personnalisation des rapports d'affaires et fournit une information utile et disponible sur les compétences existantes. La consolidation de ces rapports se réalise aussi à travers le système des tontines, lieu de convivialité et de gestion de l'information économique. Ces dernières apparaissent comme un cadre de transmission et de gestion de l'information entre entrepreneurs, à moindre coût.

Du point de vue du rôle du propriétaire, on peut souligner la mutualisation de la formation et de la professionnalisation. Celle-ci se rapporte à l'acquisition d'une expérience plus grande centrée sur une spécialité précise et bien maitrisée avec le temps. L'entrepreneur peut mieux se concentrer sur un seul domaine et créer les conditions de sa performance et de son inventivité. Il est en outre appelé à partager son expérience, à sous-traiter son savoir puis son savoir-faire pour pouvoir tirer avantage de multiples compétences détenues par d'autres acteurs au regard d'activités complémentaires. Cette gestion du long terme prend appui sur l'information et la formation tout en intégrant la prise en compte du risque, en tant que facteur de modernité. Elle participe de la recherche de mécanismes d'accroissement des compétences et de développement de capacités en fonction des besoins et objectifs que l'entreprise se fixe.

L'analyse attribue à des acteurs proactifs et interactifs ce type de changement profond ${ }^{5}$. L'engagement de l'entrepreneur bouleverse et transforme le système en modifiant la structure et les comportements (Julien et Marchesnay, 1988). La cause du changement se trouve ainsi dans la perception de l'environnement par le dirigeant et dans son style de gestion. Les mutations dans la TPE du Sud en font non plus un lieu d'obtention de rente mais un lieu de production, d'accroissement de la compétitivité, de création de richesses qui assurent un avenir moins sombre à des acteurs de plus en plus nombreux.

5. Des études sur le changement ont permis de distinguer deux types de changement. Le changement de premier ordre prend place à l'intérieur d'un système qui reste inchangé tandis que le changement de second ordre modifie le système lui-même (Guilhon, 1994).

Revue internationale P.M.E., vol. 19, nº 1, 2006

(C) 2006 - Presses de l'Université du Québec

Édifice Le Delta I, 2875, boul. Laurier, bureau 450, Québec, Québec G1V 2M2 • Tél.: (418) 657-4399 - www.puq.ca

Tiré de: Revue internationale P.M.E., vol. 19, no 1, sous la direction de Louis Raymond - PME1901N

Tous droits de reproduction, de traduction et d'adaptation réservés 
L'environnement économique a considérablement évolué, à la faveur de la crise, de la restructuration et de la privatisation des grandes entreprises publiques. La TPE se trouve désormais au cœur de l'appareil de production, avec une orientation nouvelle, un statut nouveau, soit celui d'une entreprise pensée et créée.

\section{La TPE du Sud: l'affirmation d'un statut nouveau}

L'un des facteurs susceptibles d'accélérer le changement que l'on observe à l'heure actuelle est, comme le montre l'analyse, l'apparition d'un véritable entrepreneur dans la TPE du Sud. Il convient par conséquent d'en clarifier le profil.

\subsection{L'émergence de l'entrepreneur dans la TPE du Sud}

L'émergence d'un véritable «entrepreneur» dans cette nouvelle TPE nous replace au cœur de la question de l'entrepreneur et de l'évolution des comportements qu'en présente la littérature. Le contexte de cette transformation et la place faite au capital humain en sont les fondements.

\subsubsection{Le contexte du changement}

Actuellement, la petitesse des entreprises n'est plus articulée sur leur transformation future inspirée du cycle de vie de l'entreprise comme l'ont d'abord avancé Penrose (1952) et de nombreux autres auteurs et comme l'ont ensuite mis en évidence Greiner (1972) ou encore Nelson et Winter (1982). Elle témoigne en revanche de la spécificité des systèmes de gestion (Marchesnay, 1982, 1997; Burlaud, Helfer et Marchesnay, 1991; Julien, 1994), des effets de proximité comme levier stratégique (Gervais, 1978; Burlaud, Helfer et Marchesnay, 1991; d'Amboise, 1993; Julien et Marchesnay, 1988; Torrès, 2003) et, en particulier, du rôle du capital humain dans la génération de l'innovation (Julien, 1994; Marchesnay, 1997).

En effet, l'intérêt porté à l'entrepreneur n'a cessé de croître pour en faire aujourd'hui l'acteur de l'entrepreneuriat (Julien et Marchesnay, 1996). L'entrepreneur est donc celui qui, dans son environnement, saisit les occasions d'affaires qu'il perçoit. C'est à la fois un «aventurier» conscient des risques, un «entremetteur» les assumant (Verin, 1982), un agent qui prend des décisions dominantes de la vie économique, redonnant ainsi à l'entreprise une dimension. Cette analyse se démarque de celle de l'entreprise boîte noire néoclassique où l'entrepreneur est inexistant ou passif, voué plus à s'adapter à son environnement qu'à le transformer.

Cette évolution des comportements met l'accent sur l'hétérogénéité des types d'entrepreneurs en isolant le cas particulier de l'entrepreneur du Sud. Ce dernier se distingue de l'entrepreneur innovateur nord-américain, mais aussi de l'entrepreneur en réseau japonais puis de l'entrepreneur indépendant de l'Europe du Sud (Torrès,

Revue internationale P.M.E., vol. 19, nº 1, 2006

(C) 2006 - Presses de l'Université du Québec

Édifice Le Delta I, 2875, boul. Laurier, bureau 450, Québec, Québec G1V 2M2 • Tél.: (418) 657-4399 - www.puq.ca

Tiré de: Revue internationale P.M.E., vol. 19, $\mathrm{n}^{\circ} 1$, sous la direction de Louis Raymond • PME1901N

Tous droits de reproduction, de traduction et d'adaptation réservés 
2001). Pour ces trois dernières catégories, l'un des points communs est l'implication à long terme des acteurs. Qu'il s' agisse d'une dynamique libérale, corporatiste ou de réseau, les objectifs comme les ressources sont définis dans la durée. L'entrepreneur y est un acteur «actif», au contraire de son homologue du Sud dont la production semble commandée plus par des besoins immédiats ou à très court terme que par une stratégie planifiée (Lautier, 1994; Penouil, 1992; Hernandez, 1995). Et, c'est cette dernière image que l'on retient souvent de la TPE dans les pays du Sud. L'une des explications qui peut être apportée à cette situation est la prédominance pendant longtemps dans ces pays de sa forme traditionnelle. Il apparaît pourtant que le comportement de l'entrepreneur constaté au Japon, en Amérique ou en Europe est tributaire du contexte économique, de son évolution, de la culture et, prioritairement, du rôle que doit y jouer l'entrepreneur, considéré comme une pièce maîtresse. Or, le contexte dans les pays du Sud a subi une mutation profonde. Les privatisations, les suppressions de postes dans le secteur public, les recrutements timides dans ce secteur comme dans le privé, la réduction des effectifs parmi les diplômés des universités et grandes écoles apparaissent comme le moteur de la situation dans le secteur informel de ces pays. Les «déflatés» (personnel licencié pour causes économiques) du secteur public et des grandes entreprises parapubliques et privées doivent désormais se prendre en charge, tout comme les vagues successives de diplômés qui ne sont plus absorbés automatiquement par le secteur formel de l'économie. Les effets induits de ces retraits de nombreuses activités sont la création d'occasions d'investissements offertes notamment aux cadres et autres personnels qui cherchent à rentabiliser les droits obtenus à la suite de leurs licenciements ou à tous ceux qui désirent valoriser par eux-mêmes la formation reçue. Il se constitue ainsi progressivement une nouvelle catégorie d'entrepreneurs qui confèrent à la TPE un statut nouveau.

\subsubsection{Le capital humain dans la TPE}

L'analyse établit, entre autres, une relation entre les salaires, le nombre d'années d'études et l'expérience professionnelle. Elle rattache aussi l'amélioration des conditions de travail dans le secteur informel aux succès économiques et financiers, par le biais des effets de retombées (Tidjani, 2003). La TPE désormais propulsée au cœur de l'activité doit pouvoir impulser cette force et créer ces liens. Elle cesse d'établir des relations de travail influencées par le contexte familial, où les normes, les principes puis les obligations propres à la famille sont transférés au niveau de l'entreprise. Au rôle social, celle-ci doit désormais substituer un rôle économique. En d'autres termes, elle est structurée non plus par des rapports sociaux où prévalent les stratégies de survie et de solidarité, mais par des exigences de rentabilité, de valorisation de la formation. L'acteur de ce nouvel environnement se place bien au-delà des préoccupations physiologiques, pour entrer dans une logique d'accomplissement au sens de Maslow.

Revue internationale P.M.E., vol. 19, $\mathrm{n}^{\circ} 1,2006$

(C) 2006 - Presses de l'Université du Québec

Édifice Le Delta I, 2875, boul. Laurier, bureau 450, Québec, Québec G1V 2M2 • Tél.: (418) 657-4399 - www.puq.ca

Tiré de: Revue internationale P.M.E., vol. 19, no 1, sous la direction de Louis Raymond - PME1901N

Tous droits de reproduction, de traduction et d'adaptation réservés 
L'un des déterminants de cette évolution se trouve dans le profil actuel du dirigeant fondateur de la TPE, essentiellement relié à la formation. Elle est considérée comme centrale dans la conduite du secteur informel, dans ses contraintes et préoccupations. Son existence, sa pérennité dépendent des dynamiques humaines. Le capital humain étant formé par l'ensemble des compétences, qualifications et autres capacités possédées par un individu à des fins productives, il s'acquiert par la transmission des savoirs et qualifications durant le cursus scolaire, universitaire ou au cours d'expériences professionnelles (Simonnet, 2003).

Une enquête menée au Cameroun par le Centre de recherche et d'études en économie et sondage (CRETES), au cours du deuxième semestre 2002, porte sur un échantillon de 300 très petites, petites et moyennes entreprises, toutes regroupées sous l'appellation PME, avec comme critères distinctifs le niveau de l'investissement et le nombre d'employés ${ }^{6}$. Elle porte sur plusieurs activités dont essentiellement l'agro-industrie, la boulangerie, le textile et le cuir, la transformation du bois, la fabrication chimique et électrique, le BTP et services, les services aux entreprises, les services aux ménages. L'enquête met en lumière le profil des dirigeants et leurs besoins en formation du personnel. Elle montre que les dirigeants des TPE ont de plus en plus le même profil que ceux des PE et des PME, la différence provenant surtout des ressources engagées à l'origine du projet. Pour de nombreuses activités, ils sont en majorité des diplômés de l'enseignement supérieur. Ainsi, en moyenne, $49 \%$ ont le niveau du supérieur, $39 \%$ celui du secondaire, $12 \%$ celui du primaire. Comparés aux résultats des enquêtes précédentes, notamment celle réalisée en 1994 par le même centre, le profil évolue positivement. En effet, en 1994, $42 \%$ des dirigeants avaient le niveau du supérieur, $46 \%$, celui du secondaire et $12 \%$ celui du primaire. L'amélioration se réalise au profit du supérieur ainsi qu'on peut le voir dans le tableau 2.

TABLEAU 2

Profil comparé du niveau d'instruction des dirigeants propriétaires de 300 PME au Cameroun en 1994 et 2002

\begin{tabular}{lll}
\hline \multirow{2}{*}{ Niveau d'instruction } & \multicolumn{2}{c}{ Années } \\
\cline { 2 - 3 } & $\mathbf{1 9 9 4}$ & $\mathbf{2 0 0 2}$ \\
\hline Supérieur & $46 \%$ & $49 \%$ \\
Secondaire & $42 \%$ & $39 \%$ \\
Primaire & $12 \%$ & $12 \%$ \\
\hline
\end{tabular}

Source: Constitué à partir des résultats de l'enquête du CRETES.

6. Il faut dire que l'analyse sur la petite entreprise ne s'affine que progressivement. Le terme PME semblait encore il n'y a pas très longtemps regrouper TPE, PE et PME.

Revue internationale P.M.E., vol. 19, $\mathrm{n}^{\circ}$ 1, 2006

(C) 2006 - Presses de l'Université du Québec

Édifice Le Delta I, 2875, boul. Laurier, bureau 450, Québec, Québec G1V 2M2 • Tél.: (418) 657-4399 - www.puq.ca

Tiré de: Revue internationale P.M.E., vol. 19, n 1, sous la direction de Louis Raymond • PME1901N

Tous droits de reproduction, de traduction et d'adaptation réservés 
La tendance du niveau d'instruction est intéressante dans la mesure où, d'une part, elle doit accompagner celle des activités et, d'autre part, assurer leur qualité pour une plus grande valorisation du potentiel humain et pour un redéploiement de la TPE. Elle révèle que les activités de production nécessitent souvent une formation adéquate donc plus de technicité, établissant ainsi une relation entre le profil du dirigeant et les activités.

Des études menées sur les TPE dans les pays en développement (Ferrier, 2002) dont le Swaziland, le Niger, la république du Congo, le Kenya et subsidiairement les pays d'Amérique latine (Bolivie, Colombie, Pérou) confirment cette relation. Plus le niveau d'instruction est élevé, plus la part des activités requérant plus de technicité le seront. La part des activités manufacturières dans la composition des activités des TPE sera ainsi plus importante au Swaziland que dans un pays comme le Niger. Ainsi au Swaziland, en 1991, 61\% des activités sont manufacturières, $32 \%$ commerciales et près de $7 \%$, de services. Pour le Niger, la répartition est respectivement de $21 \%, 54 \%$ et $25 \%$ environ pour l'année 1988 . Ces données sont reprises dans le tableau 3.

TABLeau 3

Structure de la formation dans les activités des TPE au Swaziland et au Niger

\begin{tabular}{lcc}
\hline \multirow{2}{*}{ Formation } & \multicolumn{2}{c}{ Répartition } \\
\cline { 2 - 3 } & Swaziland & Niger \\
\hline Études supérieures & $61 \%$ & $21 \%$ \\
Études secondaires & $32 \%$ & $54 \%$ \\
Études primaires & $7 \%$ & $25 \%$ \\
\hline
\end{tabular}

Source: Constitué à partir des données tirées de Ferrier (2002).

La structure des opérations n'est pas homogène pour toutes les économies. Son évolution est aussi fonction du taux de scolarisation et des infrastructures existantes pour assurer la formation. Globalement, ces données corroborent et complètent les résultats précédents et ceux obtenus auprès d'autres sources comme on le verra plus loin. Elles rendent compte de l'incidence du contexte sur les performances de la TPE et mettent en relief le rôle moteur du dirigeant et surtout de son profil.

\subsection{Le profil rénové de l'entrepreneur dans la TPE du Sud}

La dynamique de l'entrepreneur donne une dimension à la TPE, dimension qui est fonction du profil de l'acteur principal et de son interaction avec l'environnement. Cette relation fondamentale pour notre analyse s'appuie sur la théorie du champ de Lewin et pourrait aider à élaborer une typologie de la TPE.

Revue internationale P.M.E., vol. 19, $\mathrm{n}^{\circ} 1,2006$

(C) 2006 - Presses de l'Université du Québec

Édifice Le Delta I, 2875, boul. Laurier, bureau 450, Québec, Québec G1V 2M2 • Tél.: (418) 657-4399 - www.puq.ca

Tiré de: Revue internationale P.M.E., vol. 19, $\mathrm{n}^{\circ}$ 1, sous la direction de Louis Raymond - PME1901N

Tous droits de reproduction, de traduction et d'adaptation réservés 


\subsubsection{L'entrepreneur interactif}

L'action de l'individu se modifie en effet avec l'environnement. La théorie du champ de Lewin $(1946,1951)$ explique bien cet interactionnisme du comportement où le comportement $(\mathrm{C})$ est présenté comme une fonction $(\mathrm{F})$ de la personne $(\mathrm{P})$ et de son environnement (E). On peut donc écrire: $C=F(P, E)$.

Dans cette équation, l'état de l'individu et de son environnement sont deux facteurs interdépendants. La perception que l'on a de son environnement dépend d'un certain nombre de facteurs tels que le stade de développement, la culture, le caractère, etc. L'environnement en retour influence l'état psychologique de la personne, son action, sa réaction. Le comportement de l'entrepreneur, dans les pays du Sud comme ailleurs, doit donc nécessairement être contextualisé. L'évolution des besoins, qui suit les mutations de l'économie, impose comme partout ailleurs de nouvelles exigences à ces entreprises qui représentent l'essentiel du tissu de production. Celui-ci ne saurait se soustraire à cette «constellation de facteurs interdépendants» avec l'entrepreneur comme capital majeur. Il doit donc être apprécié différemment au fur et à mesure des changements. La place centrale de son profil, surtout sa relation avec la nature des activités transparaît dans la modification de celles-ci au fur et à mesure que le niveau d'instruction augmente.

Ainsi, parmi les 199 chefs d'entreprises formés au Cameroun par la Mipromalo $^{7}$ au cours de l'année 2002, 55\% ont obtenu un diplôme d'études supérieures, $45 \%$, un DES. Ils œuvrent en général dans les services et les activités manufacturières, en l'occurrence la fabrication de matériaux de production tels que les briques de terre, les tuiles, les carreaux, à partir principalement de matières premières locales. Les exigences de l'activité et de la formation convergent pour une maîtrise de la qualité des services, des techniques de production, de la gestion de l'entreprise. Cette interaction est illustrée par les tableaux 4 et 5 .

TABLeau 4

Relation profil-activité dans 300 PME au Cameroun

\begin{tabular}{|c|c|c|}
\hline \multirow[t]{2}{*}{ Activités } & \multicolumn{2}{|c|}{ Qualification } \\
\hline & Études supérieures & Études secondaires au plus \\
\hline Services aux entreprises & $100 \%$ & l \\
\hline Transport & $77 \%$ & $23 \%$ \\
\hline Hôtellerie et restauration & $68 \%$ & $32 \%$ \\
\hline BTP et services & $59 \%$ & $41 \%$ \\
\hline
\end{tabular}

Source: Constitué à partir des résultats de l'enquête nº 22 du CRETES.

7. La MIPROMALO est la Mission de promotion des matériaux locaux. Elle constitue une émanation du ministère de la Recherche scientifique et technique et poursuit une mission de conseil, d'aide à la création, à la formation technique et en gestion, à l'assistance dans la mise en service des installations dans les TPE et PE transformant la matière première locale.

Revue internationale P.M.E., vol. 19, n 1, 2006

(C) 2006 - Presses de l'Université du Québec

Édifice Le Delta I, 2875, boul. Laurier, bureau 450, Québec, Québec G1V 2M2 • Tél.: (418) 657-4399 - www.puq.ca

Tiré de: Revue internationale P.M.E., vol. 19, no 1, sous la direction de Louis Raymond • PME1901N

Tous droits de reproduction, de traduction et d'adaptation réservés 
TABLEAU 5

Relation profil-activité dans 199 TPE au Cameroun

\begin{tabular}{lcc}
\hline \multicolumn{1}{c}{ Types d'activités } & \multicolumn{2}{c}{ Qualification } \\
\cline { 2 - 3 } & Études supérieures & Études secondaires au plus \\
\hline $\begin{array}{l}\text { Technique de production et } \\
\text { gestion de l'entreprise }\end{array}$ & $55 \%$ & $45 \%$ \\
\hline $\begin{array}{l}\text { Source: Constitué à partir des données de la MIPROMALO, Service de la formation, Rapport d'activités } \\
\text { au 31 décembre 2002. }\end{array}$
\end{tabular}

au 31 décembre 2002.

Si le tableau 4 concerne la petite entreprise au sens large, le tableau 5 se rapporte uniquement à la TPE.

Dans la majorité de ces TPE, les proriétaires se recrutent parmi les ingénieurs, les techniciens supérieurs, les architectes, les comptables, les diplômés des écoles et instituts de gestion, etc. Ils investissent prioritairement dans les activités manufacturières et de services. Il se confirme ainsi cette relation forte entre l'environnement et le comportement des acteurs, mais qui ne traduit nullement une homogénéité entre les pays ni à l'intérieur des pays.

\subsubsection{L'hétérogénéité de la TPE}

L'hétérogénéité est effective et révélatrice d'une coexistence de plusieurs modèles de TPE avec des activités et des profils distincts, des référentiels et des modes de mesure conséquents.

L'interaction du profil de l'acteur principal avec son environnement permet de regrouper les TPE en deux grands groupes, les TPE du secteur informel traditionnel et les TPE du secteur informel moderne. Le premier groupe est constitué de la petite activité marchande tandis que le second vise les activités manufacturières. Le commerce et les services peuvent à la fois appartenir au secteur traditionnel et au secteur moderne selon le profil du propriétaire et la formation reçue. Cette typologie est présentée dans le tableau 6 .

Cette difficulté à classer correctement les activités de la TPE témoigne de la complexité du secteur informel. Néanmoins, les mutations s'affirment avec le temps et pourraient s'accélérer si les efforts en cours pour aplanir un certain nombre d'obstacles sont poursuivis. Il s'agit principalement de poursuivre la réflexion sur un des modes de gestion de l'informel qui ne dénaturent pas le secteur. 
TABLEAU 6

Quelques modèles de TPE dans les pays du Sud

\begin{tabular}{|c|c|c|c|c|}
\hline & $\begin{array}{c}\text { Identification } \\
\text { des TPE }\end{array}$ & $\begin{array}{c}\text { Profil de } \\
\text { l'entrepreneur }\end{array}$ & Référentiel & $\begin{array}{c}\text { Mode } \\
\text { de mesure }\end{array}$ \\
\hline \multirow{2}{*}{$\begin{array}{l}\text { Secteur } \\
\text { informel } \\
\text { traditionnel }\end{array}$} & $\begin{array}{l}\text { Petite activité } \\
\text { marchande. }\end{array}$ & $\begin{array}{l}\text { Formation } \\
\text { sur le tas. }\end{array}$ & Survie. & $\begin{array}{l}\text { Comportement } \\
\text { de subsistance. }\end{array}$ \\
\hline & Commerce & $\begin{array}{l}\text { Formation mixte } \\
\text { avec formation } \\
\text { sur le tas } \\
\text { dominante. }\end{array}$ & $\begin{array}{l}\text { Complexe: } \\
\text { - proximité, } \\
\text { - création de } \\
\text { richesses. }\end{array}$ & $\begin{array}{l}\text { Comportement: } \\
\text { - de subsistance, } \\
\text { - d'investissement } \\
\text { dans } \\
\text { l'immobilier } \\
\text { et autres. }\end{array}$ \\
\hline \multirow[t]{2}{*}{$\begin{array}{l}\text { Secteur } \\
\text { informel } \\
\text { moderne }\end{array}$} & Services & $\begin{array}{l}\text { Formation mixte } \\
\text { avec formation } \\
\text { secondaire et } \\
\text { universitaire } \\
\text { dominante. }\end{array}$ & $\begin{array}{l}\text { Originalité: } \\
\text { - personnalisa- } \\
\text { tion } \\
\text { du service, } \\
\text { - service de } \\
\text { proximité. }\end{array}$ & $\begin{array}{l}\text { Comportement de } \\
\text { complémentarité } \\
\text { et d'affirmation } \\
\text { personnelle. }\end{array}$ \\
\hline & $\begin{array}{l}\text { Activités } \\
\text { manufacturières }\end{array}$ & $\begin{array}{l}\text { Formation } \\
\text { essentiellement } \\
\text { secondaire et } \\
\text { universitaire. }\end{array}$ & $\begin{array}{l}\text { Implication à } \\
\text { long terme: } \\
\text { - originalité, } \\
\text { - pérennité. }\end{array}$ & $\begin{array}{l}\text { Comportement } \\
\text { d'accomplissement } \\
\text { et de valorisation } \\
\text { de la formation. }\end{array}$ \\
\hline
\end{tabular}

Source: Constitué à partir des données de l'analyse.

\section{Conclusion}

Le changement intervenu aujourd'hui dans l'environnement socioéconomique des entreprises du fait des grandes mutations, parmi lesquelles les privatisations, amène de nombreuses économies du Sud à s'interroger sur ce que pourrait être un mode plus dynamique de fonctionnement de la TPE. Une telle préoccupation pour l'avenir de la petite entreprise n'est ni nouvelle ni localisée. On la retrouve en Europe, notamment face à l'échéance de 1993. Elle s'est d'abord traduite dans une crainte suivie d'une réflexion quant au sort de la petite entreprise. Elle s'est concrétisée ensuite dans la mise en œuvre de dispositifs pour protéger et renforcer la position concurrentielle des firmes de petite taille face à des géants européens s'organisant pour résister aux groupes du reste du monde. Elle est d'ailleurs loin d'être dissipée.

S'agissant de la TPE du Sud, elle n'est pas une nouveauté, étant même l'activité de production traditionnelle. Toutefois, sa forme n'est pas restée figée. Cette transformation, incontournable au regard des contraintes qu'elle doit desserrer, est justifiée pour plusieurs raisons. Elle permet d'abord de se rendre compte que si le problème est posé globalement, les réponses ne sauraient être identiques pour

Revue internationale P.M.E., vol. 19, n 1, 2006

(C) 2006 - Presses de l'Université du Québec

Édifice Le Delta I, 2875, boul. Laurier, bureau 450, Québec, Québec G1V 2M2 • Tél.: (418) 657-4399 - www.puq.ca

Tiré de: Revue internationale P.M.E., vol. 19, $\mathrm{n}^{\circ} 1$, sous la direction de Louis Raymond • PME1901N

Tous droits de reproduction, de traduction et d'adaptation réservés 
l'ensemble des économies, pour toutes les TPE, puisqu'il ne s'agit nullement d'un ensemble homogène. Elle exige ensuite de prendre conscience de l'importance des enjeux, des occasions d'affaires qui s'offrent à la TPE et des dangers qu'elle encourt si les conditions pour saisir et exploiter au mieux ces chances ne sont pas créées. Par conséquent, si les enjeux sont importants et les opportunités, nombreuses, il est urgent que la TPE soit préparée à la gestion de ce nouveau contexte, c'est-à-dire à la gestion de l'informel.

Paradoxalement, on doit reconnaitre que l'informel qui entoure la création et le développement de la TPE est une des raisons de son existence et apparaît tantôt comme un frein, tantôt comme un levier pour des choix immédiats ou futurs plus ambitieux. Dans ce sens, deux directions au moins sont envisageables. La gestion de l'informel passe par un système d'identification et de fiscalisation du secteur ou alors par l'allègement des formalités de passage au secteur formel, pour les TPE qui le souhaitent.

Ainsi, la facilité d'entrée dans l'économie informelle est présentée comme une force, tenue d'ailleurs pour une des constantes des analyses des organisations internationales. Cette perméabilité du secteur informel est liée essentiellement au niveau de ressources nécessaires pour créer une TPE. Ces ressources sont facilement accessibles pour les individus ou les familles engagées dans cette logique, au-delà de la barrière à son développement que constitue leur modicité. Le développement implique de ce fait la diversification de l'origine des ressources pour accroître durablement le niveau et la qualité de celles-ci afin de mieux les intégrer dans une dynamique de rentabilité, de croissance et de pérennité. C'est un axe indispensable pour consolider l'action de la TPE, celle du XXI ${ }^{e}$ siècle qui doit passer de la satisfaction des besoins d'une économie de subsistance à celle d'une orientation sur le long terme.

En tant que faiblesse, l'une des principales caractéristiques de ces unités de production est l'absence d'une existence juridique systématique. La TPE tend à fonctionner sans règles formelles de droit. La petite taille des entreprises, l'éparpillement et la spécificité des compétences puis la complémentarité qu'impose la fabrication d'un produit ou l'offre d'un service requièrent pourtant l'existence d'un mode précis de coordination des activités. Dans cet univers de relations informelles où tout contrat est tacite ou implicite, celles-ci sont compensées par le développement d'un capital de relations personnelles et informelles qui dominent la réalité quotidienne et fait naître un certain nombre d'obligations réciproques. Or, le rapport à la loi est un aspect majeur pour penser la dynamique de l'économie, même informelle. Pour y parvenir, il est important d'examiner la question du passage au formel. La solution, semble-t-il, ne réside pas dans l'éradication de ces dernières activités. De même, leur tolérance par les pouvoirs publics montre de plus en plus ses limites. La fiscalisation du secteur s'impose ainsi de plus en plus comme la

Revue internationale P.M.E., vol. 19, $\mathrm{n}^{\circ} 1,2006$

(C) 2006 - Presses de l'Université du Québec

Édifice Le Delta I, 2875, boul. Laurier, bureau 450, Québec, Québec G1V 2M2 • Tél.: (418) 657-4399 - www.puq.ca

Tiré de: Revue internationale P.M.E., vol. 19, no 1, sous la direction de Louis Raymond - PME1901N

Tous droits de reproduction, de traduction et d'adaptation réservés 
voie de la raison. L'allégement des délais et des formalités de «légalisation», la création de centres uniques de formalités sont proposés comme des instruments de cette politique pour les TPE qui veulent franchir ce cap. Ce dispositif peut constituer le point de départ de relations de coopération durables entre entreprises nationales, régionales et sous-régionales. L'harmonisation du droit des affaires en Afrique avec l'entrée en vigueur depuis le $1^{\text {er }}$ janvier 2001 de l'Organisation pour l'harmonisation en Afrique du droit des affaires y apporte une impulsion. La dynamique de la TPE reste en mouvement et requiert une réflexion permanente pour le renforcement des voies et des moyens.

\section{Bibliographie}

BARREDY, C. (2002), «Structures juridiques, gouvernance et développement de l'entreprise», dans J. Caby et G. Hirigoyen (dir.), La Gestion des entreprises familiales, Paris, Economica, p. 127-147.

BASLY, S. (2002), «L'internationalisation de l'entreprise familiale» dans J. Caby et G. Hirigoyen (dir.), La Gestion des entreprises familiales, Paris, Economica, p. 149-173.

Batsch, L. (1997), «Temps et gestion», dans P. Joffre et Y. Simon (dir.), Encyclopédie de gestion, tome 3, Paris, Economica, p. 3303-3309.

BIT (1972), Employment, Incomes and Equality: A Strategy for Increasing Productive Employment in Kenya, Genève, Publications de l'OIT.

Burlaud, A., J.-P. Helfer et M. Marchesnay (dir.) (1991), Identités de la gestion, Mélanges en l'honneur du professeur P. Lassegue, Paris, Vuibert Gestion.

Caby, J. et G. Hirigoyen (2001), La création de valeur de l'entreprise, $2^{\mathrm{e}}$ éd., Paris, Economica.

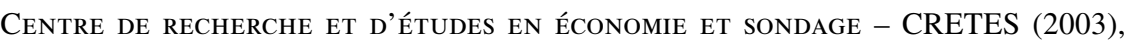
Conjoncture PME, Enquête $n^{\circ} 22$, février.

Cocula, F. (2002), Introduction générale à la gestion, Paris, Dunod.

D'Amboise, G. (1993), «Empirical research on SME's: the past ten years in Canada», Journal of Small Business \& Entrepreneurship, vol. 10, $\mathrm{n}^{\circ} 2$.

De Sото, H. (1994), L'autre sentier, la révolution informelle dans le Tiers-Monde, traduction de M. Couderc, Paris, La Découverte.

Ferrier, O. (2002), Les très petites entreprises, Bruxelles, De Boeck Université.

Fonds nATIONAL DE L'EMPloi - FNE (1999), Les Actes du $1^{\text {er }}$ Forum national de l'emploi, Douala, 18-19 mars.

Gervais, M. (1978), «Pour une théorie de l'organisation PME», Revue française de gestion, $\mathrm{n}^{\circ} 15$, p. $37-49$.

GNANSOUNOU, S. (1992), «L'épargne informel et le financement de l'entreprise productive: référence spéciale aux tontines et à l'artisanat béninois», Revue internationale PME, vol. $5, \mathrm{n}^{\text {os }} 3-4$.

Revue internationale P.M.E., vol. 19, $\mathrm{n}^{\circ} 1,2006$

(C) 2006 - Presses de l'Université du Québec

Édifice Le Delta I, 2875, boul. Laurier, bureau 450, Québec, Québec G1V 2M2 • Tél.: (418) 657-4399 - www.puq.ca

Tiré de: Revue internationale P.M.E., vol. 19, n 1, sous la direction de Louis Raymond • PME1901N

Tous droits de reproduction, de traduction et d'adaptation réservés 
GreINER, L. (1972), «Evolution and revolution as organisation grows», Harvard Business Review, vol. 50, p. 37-46.

GuILhon, B. (1994), «PME-PMI et économie industrielle», Revue d'économie industrielle, numéro spécial, $\mathrm{n}^{\circ} 67,1^{\text {er }}$ trismestre.

HERNANDEZ, E.M. (1995), «La logique de gestion de l'entreprise du secteur informel», Revue française de gestion, $\mathrm{n}^{\circ} 103$, mars-avril-mai.

HERNANDEZ, E.M. (1997), «L'État et l'entrepreneur en Afrique», Revue internationale $P M E$, vol. 10, no 1 p. 103-122.

HernANDEZ, E.M. (1997), Le management des entreprises africaines, Paris, L'Harmattan.

Hirigoyen, G. (dir.) (2002), La gestion des entreprises familiales, Paris, Economica.

Julien, P.-A. (1994), Les PME. Bilan et perspectives, Paris, Economica; Québec, Presses Inter Universitaires.

Julien, P.-A. et M. Marchesnay (1988), La petite entreprise, Paris, Vuibert Gestion.

Julien, P.-A. et M. Marchesnay (1996), L'Entrepreneuriat, Paris, Economica.

Kengne, F. et A. Metton (2000), Économie informelle et développement dans les pays du Sud à l'ère de la mondialisation, Yaoundé, Presses universitaires de Yaoundé, p. 25-26.

Knight, F. (1921), Risk, Uncertainty and Profit, New York, Houghton Mifflin.

LAutier, B. (1994), L'économie informelle dans le Tiers-Monde, Paris, La Découverte, Repères.

LEwIN, K. (1946), «Behaviour and development as a function of the total situation», dans L. Carmichael (dir.), Manual of Child Psychology, New York, John Wiley and Sons. Réédité en 1997 dans K. Lewin (dir.), Resolving Social Conflicts \& Field Theory in Social Science, Washington, American Psychological Association, p. 337-381.

Lewin, K. (1951), Field Theory in Social Science, New York, Harper \& Row.

Loup, S. (2003), «Les petites entreprises des métiers d'art», Revue française de gestion, vol. 29, n 144, mai-juin, p. 195-209.

Marchesnay, M. (1982), Pour un modèle d'hypofirme, Paris, Economica, coll. «Entreprises et organisations».

Marchesnay, M. (1997), «Petite entreprise et entrepreneur», dans Y. Simon et P. Joffre (dir.), Encyclopédie de gestion, Paris, Economica, p. 2209-2219.

Marchesnay, M. (2003), «La petite entreprise: sortir de l'ignorance», Revue française de gestion, vol. 29, n 144, mai-juin, p. 107-118.

Marwaha, M.G. (2002), «Innovative financing and credit systems for SMEs», World Convention of SMEs at Changzhou, Chine, septembre.

Nelson, R.R. et S.G. Winter (1982), An Evolutionary Theory of Economic Change, Cambridge, Belknap Press of Harvard University Press.

Ntsame, E., B. Bekolo-Ebe, M.-T. Um-Ngouem, M. Ndong-Ntah et L.M. Abessolo (1999), Élaboration d'une stratégie de financement et de garantie des crédits aux PME au Cameroun, Rapport d'étude MINDIC/PNUD.

Revue internationale P.M.E., vol. 19, $\mathrm{n}^{\circ} 1,2006$

(C) 2006 - Presses de l'Université du Québec

Édifice Le Delta I, 2875, boul. Laurier, bureau 450, Québec, Québec G1V 2M2 • Tél.: (418) 657-4399 - www.puq.ca

Tiré de: Revue internationale P.M.E., vol. 19, no 1, sous la direction de Louis Raymond • PME1901N

Tous droits de reproduction, de traduction et d'adaptation réservés 
Ntsama, E., M.-T. Um-Ngouem et L.M. Abessolo (2003), Définition de la PME, de la micro-entreprise et de l'artisanat au Cameroun, Rapport d'études commandé par MINDIC/AFD.

PACITTO, J.-C. (1998), «Quel marketing pour les très petites entreprises?», Revue française de gestion, $\mathrm{n}^{\circ} 121$, nov.-déc., p. 42-52.

Penouil, M. (1992), «L'avenir de l'entreprise africaine», dans Mélanges en l'honneur du professeur J.G. Merigot, Paris, Economica.

Penrose, E. (1952), «Biological analogies in the theory of the firm», American Economic Review, vol. 42.

Rofe, N. (1998), Étude de faisabilité du dispositif d'appui et de financement des TPE au Cameroun, Rapport d'étude, février.

SAPorta, B. et L. Kombou (2000), Histoire d'entreprendre: les réalités de l'entrepreneuriat, Colombelles, Éditions EMS, Management et Sociétés.

SAPORTA, B. (2002), «Famille, création d'entreprises et entrepreneuriat», dans J. Caby et G. Hirigoyen (dir.), La Gestion des entreprises familiales, Paris, Economica, p. 107-125.

Sime Zadouo, M. (2003), «Profil des dirigeants des PME et besoins en formation du personnel», dans l'enquête $\mathrm{n}^{\circ} 22$ du CRETES, Conjoncture PME, février.

Simonnet, V. (2003), «Le capital humain», dans Encyclopédie des ressources humaines, Paris, Vuibert, p. 133-144.

Srinivasan, N. et J. Mammen (2002), «Role of small business in promoting rural industrialisation», SME Leaders, Workshop Towards WASME Conference 2003, 7-14 décembre, Tel Aviv.

Tidjani, B. (2003), «La gestion des ressources humaines dans le secteur informel en Afrique», dans Encyclopédie des ressources humaines, Paris, Vuibert, p. 19-31.

Torrès, O. (dir.) (1998), PME, de nouvelles approches, Paris, Economica.

Torrès, O. (2001), «Les divers types d'entrepreneuriat et de PME dans le monde», Management international, vol. $6, \mathrm{n}^{\circ} 1$.

TORRÈs, O. (2003), «Petitesse des entreprises et grossissement des effets de proximité», Revue française de gestion, vol. 29, nº 144, mai-juin, p. 119-138.

Um-Ngouem, M.-T. (1996), Financement bancaire et gestion des PME camerounaises, Thèse de doctorat, Université de Montesquieu, Bordeaux IV, février.

Um-Ngouem, M.-T. (1997), «La spécificité du problème de l'investissement dans les PME camerounaises », dans Notes de recherches de l'AUPELF-UREF, $\mathrm{n}^{\text {os }}$ 97-61.

Verin, H. (1982), Entrepreneurs, entreprises. Histoire d'une idée, Paris, Presses universitaires de France.

World SME News (2002), Publications de la World Association for Small and Medium Enterprises, $\mathrm{n}^{\circ} 3$, octobre-novembre.

Revue internationale P.M.E., vol. 19, nº 1, 2006

(C) 2006 - Presses de l'Université du Québec

Édifice Le Delta I, 2875, boul. Laurier, bureau 450, Québec, Québec G1V 2M2 • Tél.: (418) 657-4399 - www.puq.ca

Tiré de: Revue internationale P.M.E., vol. 19, no 1, sous la direction de Louis Raymond • PME1901N

Tous droits de reproduction, de traduction et d'adaptation réservés 